\title{
Potential role of daratumumab in the treatment of multiple myeloma
}

\author{
Yulian Khagi' \\ Tomer M Mark ${ }^{2}$ \\ 'Department of Medicine, New York \\ Presbyterian Hospital-Cornell Medical \\ Center, New York, NY, USA; '2Division \\ of Hematology and Medical Oncology, \\ Department of Medicine, Weill \\ Cornell Medical College, New York \\ Presbyterian Hospital-Cornell Medical \\ Center, New York, NY, USA
}

Correspondence: Tomer M Mark Department of Medicine, New York Presbyterian Hospital-Cornell Medical Center, 525 East 68th Street,

New York, NY 1002I, USA

$\mathrm{Tel}+\mathrm{I} 2$ I 27463964

Fax + I 2 I2 746896 I

Email tom9009@med.cornell.edu

\author{
This article was published in the following Dove Press journal: \\ OncoTargets and Therapy \\ 18 June 2014 \\ Number of times this article has been viewed
}

\begin{abstract}
Multiple myeloma is the second most common hematologic malignancy in the US. Treatments utilizing alkylating agents, corticosteroids, proteasome inhibitors, and immunomodulatory drugs have resulted in significant survival benefits, however, despite the advances, relapse is inevitable. Decreased depth and duration of response obtained with each successive relapse of disease is typical of the disease course, thereby highlighting a continuing need for new treatment options. With the introduction of monoclonal antibodies for multiple myeloma, new options for treatment in the relapsed setting are on the horizon. Among the new immunologic agents is daratumumab (DARA), a humanized antibody to CD38 with potent multifaceted antitumor activity. Phase I and II clinical trials have demonstrated significant reduction in serum M-protein and bone marrow plasma cell percentage in refractory patients, with an acceptable toxicity profile. Moreover, ex vivo studies have shown that DARA may be particularly useful in combination with currently used anti-myeloma agents. With a recent breakthrough drug designation by the US Food and Drug Administration, DARA shows promise as mono- and combination therapy for the treatment of relapsed/refractory multiple myeloma.
\end{abstract}

Keywords: multiple myeloma, relapsed, refractory, monoclonal antibody, daratumumab, CD38

\section{Introduction}

Multiple myeloma (MM) accounts for approximately $10 \%$ of newly diagnosed hematologic malignancies in the US and is characterized by the proliferation of malignant clonal plasma cells that produce a single immunoglobulin isotype, called an M-protein. ${ }^{1}$ Clonal expansion within bone marrow and tissue leads to bone destruction, hypercalcemia, marrow dysfunction, frequent infections, as well as renal failure. ${ }^{2}$ Prior to the advent of proteasome inhibitor and immunomodulator-based therapy, when alkylating chemotherapy with corticosteroids was standard of care, survival was for three decades. ${ }^{3}$ The proteasome inhibitor bortezomib (BORT) and the immunomodulatory drugs thalidomide and lenalidomide induce impressive responses with improvement in overall survival, yet relapse remains inevitable, usually with disease refractory to currently available therapies. ${ }^{4}$ Notably, patients who have disease relapse after treatment with lenalidomide and BORT have a particularly poor prognosis, with median overall survival of nine months. ${ }^{5}$

In July 2012, carfilzomib became the newest proteasome inhibitor to be US Food and Drug Administration (FDA) approved for relapsed/refractory multiple myeloma (RRMM). ${ }^{6}$ In February 2013, pomalidomide was approved as the next immunomodulatory drug. The two agents have been shown to be both safe and efficacious in the treatment of heavily pre-treated RRMM, including patients who have relapsed after 
prior BORT and/or lenalidomide therapy. ${ }^{7-10}$ Yet, even with encouraging response data and improved progression free survival (PFS), resistance develops rapidly and PFS for patients receiving either of these two agents remains short, in the order of 4-8 months. ${ }^{11,12}$ Clearly, new effective agents are needed for the management of RRMM galvanizing the search for an immunologic approach similar to the successes seen in B-cell lymphomas.

\section{Monoclonal antibodies in multiple myeloma}

Until recently, monoclonal antibody therapies have not yielded success in the treatment of MM. With the introduction of rituximab, a humanized monoclonal antibody against CD20, as the first FDA-approved monoclonal antibody for lymphoma treatment in 1997, the oncologic community has seen remarkable outcomes spanning several hematologic malignancies. Disappointingly, with regards to MM, two Phase II studies of rituximab have shown overall response rates of only $0 \%-5 \% .{ }^{13,14}$ The reason for this was thought to be due to only a minority of MM clones expressing the target CD20 antigen. ${ }^{15}$ Nonetheless, it was discovered that even in that minority of specimens that were densely CD20 positive, responses were still poor. ${ }^{16}$

Two other agents tested for myeloma include elotuzumab (ELO) and milatuzumab. ${ }^{17-20}$ ELO is a humanized monoclonal IgG1 antibody that targets CS-1, a cell surface glycoprotein with dense expression in malignant plasma cells. Targeting CS-1 has been shown to lead to natural killer (NK)-cell mediated antibody dependent cellular cytotoxicity (ADCC). ${ }^{17,19}$ Clinical trials have combined ELO with lenalidomide (LEN) and dexamethasone (DEX) in patients with relapsed myeloma. The results of these studies have been promising with $90 \%$ of patients achieving partial response (PR) and PFS of $>2$ years. ${ }^{19,20}$ A Phase III study is ongoing and due to be completed in $2017 .{ }^{20}$ It is important to note, however, that there have been no clinical trials demonstrating single agent efficacy with ELO and that a Phase I single agent dose escalation study from 2012 demonstrated no objective response. ${ }^{21}$

Another agent currently under investigation for the treatment of MM (as well as non-Hodgkin's lymphoma) is milatuzumab, a selective humanized antibody targeting CD74. ${ }^{18}$ CD74 is a component of the major histocompatibility complex class II complex that is heavily expressed in B-cell neoplasms and found only marginally in normal tissue. CD74 expression has been demonstrated in up to $95 \%$ of MM samples. ${ }^{22,23}$ Preclinical studies have shown that not only can the antibody induce apoptosis when bound to its target, but also that the rapid internalization and recycling of CD74 makes it possible to conjugate chemotherapeutics, radioisotopes and other toxins to the drug to make targeted, efficacious killing a possibility. ${ }^{24-30}$ However, clinical trials using milatuzumab in MM have yet to demonstrate significant activity. ${ }^{31}$

\section{Daratumumab}

Daratumumab (DARA) is a humanized antibody against CD38, a cell surface glycoprotein that is strongly expressed in MM. ${ }^{32,33}$ It has received breakthrough therapy designation from the FDA for encouraging activity as a single-agent in a Phase I study for RRMM. ${ }^{34}$ The biochemical, immunologic, and pharmacologic properties of DARA as well as the current clinical data supporting its use are discussed below.

\section{Synthesis and selection}

DARA was selected from 42 candidate antibodies generated from immunization of transgenic mice with recombinant CD38 protein. ${ }^{33}$ Its capacity to induce cytotoxicity as measured by flow cytometry in purified patient MM cells was the basis for its selection for further development. Overexpression of CD38 is seen on most malignant plasma cells in all stages of multiple myeloma. ${ }^{35}$ Moreover, MM plasma cell precursors are highly CD38 positive, indicating that the target may be highly expressed in MM stem cells. ${ }^{36,37}$

\section{Function of CD38}

CD38 is involved in the regulation of cell adhesion by receptor-mediated intracellular signaling. Its other function is as an ecto-enzyme to catalyze metabolism of cyclic adenosine diphosphate-ribose and nicotinic acid adenine dinucleotide phosphate. ${ }^{38}$ This metabolism is key for the regulation of calcium messaging within the endoplasmic reticulum and lysosomal calcium stores. ${ }^{39,40}$ Additionally, CD38 signaling also involves cross-talk with antigen-receptor complexes on $\mathrm{T}$ and $\mathrm{B}$ cells and is involved in switching and secretion of IgG1 as well as activation of NK cells. ${ }^{41-43}$

\section{Preclinical data}

DARA has been shown to be effective in vitro by killing $\mathrm{CD} 38^{+} \mathrm{CD} 138^{+} \mathrm{MM}$ cells via four proposed mechanisms. The first two, ADCC and complement-mediated cytotoxicity (CDC), are induced in the bone marrow microenvironment and do not appear to be influenced by surrounding stromal cells. ${ }^{33,44}$ The third mechanism by which DARA can lead to cell death is by directly inducing cytotoxicity through 
cross-linkage of DARA with anti-human IgG antibody and Fc receptors expressed on effector cells. ${ }^{45}$ The fourth mechanism of action is by inducing cell death via antibody-dependent cellular phagocytosis (ADCP). Akin to opsonization, ADCP is achieved by binding of Fc receptors on NK cells and macrophages by tumor-bound DARA. This directly signals phagocytosis of the tumor cell. ${ }^{46}$ The pharmacodynamics of DARA estimates a half-life in the serum of approximately 21 days, which is similar to other monoclonal antibodies, such as rituximab. The route of elimination at low doses of DARA is target-mediated clearance by binding to the tumor cells. ${ }^{34}$ Potential drug interactions are unknown at this time.

Interestingly, lenalidomide, as well as BORT and melphalan have enhanced MM cytotoxicity in the presence of DARA. ${ }^{47}$ Pretreatment with lenalidomide resulted in improved DARA-induced ADCC in MM cells by activating NK cells in several studies. ${ }^{47-49}$ This is discussed further in the Clinical data section below.

\section{Clinical data}

Based on preclinical data, DARA underwent a large two-part Phase I/II study for patients with heavily pre-treated RRMM who had received two or more prior lines of therapy. ${ }^{34,50,51}$ In this study, ten cohorts involving a total of 32 patients, were treated with a standard $3+3$ dose escalation scheme, from $0.005 \mathrm{mg} / \mathrm{kg}$ to $24 \mathrm{mg} / \mathrm{kg}$, administered intravenously once weekly. All cohorts were treated with two test doses (10\% of the full dose), and subsequently seven full doses for a total of 9 weeks. Median cohort age range was 55 to 64 years, median number of prior treatments was $5.5,75 \%$ of patients had been refractory to both lenalidomide and BORT, and $83 \% / 33 \%$ had undergone antecedent autologous stem cell transplantation/allogeneic transplant, respectively.

Pharmacokinetic analysis was done by enzyme-linked immunosorbent assay (ELISA) to measure DARA plasma concentration. Plasma concentration peak levels showed rapid clearance at low doses $(\leq 2 \mathrm{mg} / \mathrm{kg})$ and those clearance rates decreased with increasing dose. This was suggestive of doserelated target-mediated clearance. At doses of $\geq 4 \mathrm{mg} / \mathrm{kg}$, trough levels were sustained at $>10 \mathrm{mcg} / \mathrm{mL}$ indicative of target binding. At those levels, observed compared to predicted pharmacokinetic values were similar. ${ }^{34,50}$

Response was then measured by change in serum and urine M-protein, change in free light chain ratio and reduction in bone marrow plasma cells. There was greater M-protein reduction in cohorts treated with doses of $4 \mathrm{mg} / \mathrm{kg}$ or above. M-protein and free-light chain reduction responses were durable to at least 28 weeks. Ten out of 32 patients had clinical response,
5 out of $32(15.5 \%)$ with PR and 5 out of 32 (15.5\%) with minimal response (MR). At doses of $\geq 4 \mathrm{mg} / \mathrm{kg}$ (12 patients), 5 achieved PR (42\%) and 3 achieved MR (25\%). PFS correlated with longer exposure time and higher dose of the study drug, with approximately $70 \%$ alive without progression at about 6 months in the $\geq 4 \mathrm{mg} / \mathrm{kg}$ cohort (with median follow up 18.4 weeks) versus approximately $20 \%$ at about 6 months in the $\leq 2 \mathrm{mg} / \mathrm{kg}$ cohort (with median follow up of 8.6 weeks). ${ }^{34,50,51}$ Of note, the study was not powered for overall survival and median PFS in the $\geq 4 \mathrm{mg} / \mathrm{kg}$ groups were not reached.

Following the study of single-agent DARA in RRMM, several combination therapies using DARA with other agents for myeloma are currently underway. ${ }^{47-49,52-54}$ Van der Veer et al demonstrated in a 2011 study that clearance of MM cells is enhanced with the combination of LEN and DARA due to LEN's ability to activate T and NK cells and DARA's ability to synergistically mediate lysis by ADCC. ${ }^{49}$ An ex vivo study of drug combinations with DARA showed that while LEN alone, BORT alone, and a combination of the two were able to produce only modest lysis $(10 \%, 18 \%$, $25 \%$, respectively), the addition of DARA improved lysis by greater than two-fold in all combinations. Furthermore, addition of DARA to combination LEN, BORT, DEX (RVD) and melphalan, prednisone, BORT (MPV) resulted in nearly double the lysis seen without DARA. ${ }^{47}$

At the 2013 American Society of Hematology meeting, several abstracts had further supported the use of DARA with combination therapy. ${ }^{53}$ In one study, bone marrow plasma cells from LEN and LEN/BORT refractory patients were exposed to DARA alone or in combination with LEN/BORT. As expected, LEN and BORT alone were not able to induce significant lysis. With the addition of DARA, however, substantial levels of lysis were achieved. LEN/DARA-mediated lysis occurred in a synergistic activation of effector cells and BORT/DARA-mediated lysis increased in an additive manner. In a Phase I/II open-label multicenter study of DARA combined with LEN/DEX with a $3+3$ dose-escalated design, efficacy was shown with the addition of DARA resulting in marked reduction in M-protein, yielding six of six patients with at least PR and three of six with at least very good partial remission. ${ }^{54}$ Of note all study participants had prior treatment with lenalidomide and later had progression of disease, with the exception of the cohort receiving $<1 \mathrm{mg} / \mathrm{kg}$ of the study drug, in which $88 \%$ had been REV exposed. Safety data showed a manageable toxicity profile consistent with an LEN/DEX regimen, consisting mainly of myelosuppression. Another abstract outlined a unique approach utilizing DARA with pre-targeted radio-immunotherapy in MM xenograft 
mouse models. ${ }^{55}$ This combination showed enhanced delivery of therapeutic doses of radiation via high avidity binding of anti-CD38 antibody-tumor conjugates, and a reported induction of $100 \%$ remission rates in less than 40 days. Long-term toxicity is to be determined in this population.

\section{Safety profile}

In the Phase I/II study, the most common adverse event was infusion reaction. ${ }^{34,50}$ Infusion reactions occurred in $30 \%$ of patients during the first full infusion and in 10\% during the test doses. Infusion reactions incrementally decreased during subsequent doses after the second full infusion. Most of the infusion reactions occurred within 3-4 hours and after the addition of pre-infusion steroid (prednisolone/methylprednisolone at a maximum corticosteroid dose equivalence of $27 \mathrm{mg}$ DEX per week), no further serious infusion reactions were reported in the $\geq 4 \mathrm{mg} / \mathrm{kg}$ cohorts. Reported serious adverse events were limited to one patient of 32 in each of the following categories: grade 3 anemia, grade 4 thrombocytopenia, grade 3 rise in aspartate aminotransferase, and grade 2 cytokine release syndrome. Two patients of 32 had experienced grade 2 and 3 bronchospasm, respectively. ${ }^{34,50,51}$

Results from the Phase I portion of the study provided evidence of an acceptable safety profile and suggested significant antitumor activity in the heavily pre-treated MM population. After review of this data, the US FDA designated DARA as a "breakthrough therapy". As part of this ongoing Phase I study, doses of $8 \mathrm{mg} / \mathrm{kg}$ and $16 \mathrm{mg} / \mathrm{kg}$ are being further investigated. Additionally, a longer treatment period of 96 weeks is being used for responding patients. One of several Phase II studies will also evaluate the monotherapeutic activity of DARA in myeloma that has progressed after both prior immunomodulatory and proteasome inhibitor treatment. ${ }^{52}$

\section{A comment on future directions}

As the number of survivors with myeloma grows, so too does the need for new regimens to treat relapse of chemoresistant disease. Pomalidomide and carfilzomib have been highly successful for the treatment of RRMM, however, responses tend to be short-lived and they carry the toxicity profiles of the immunomodulatory drugs and proteasome inhibitors, respectively. DARA represents a tolerable and effective single-agent treatment option for patients with advanced RRMM, who may have significant comorbidities, such as peripheral neuropathy or myelosuppression, and who may not otherwise tolerate pomalidomide, carfilzomib, or other chemotherapy. For induction or salvage chemotherapy in fitter patients, we speculate that DARA will be added to existing regimens to enhance efficacy, analogous to the historical addition of rituximab to B cell lymphoma chemotherapy. Perhaps most excitingly, DARA may find use in halting or slowing the progression of smoldering myeloma to active myeloma without exposing otherwise asymptomatic patients to the potential toxicities of chemotherapy or immunosuppressive drugs. Further clinical studies with DARA are of course needed to provide the data to support our conjectures above.

In addition to impressive preclinical and early clinical data suggesting the benefits of DARA in the MM population, there is now preclinical data suggesting the use of DARA in other hematologic malignancies. The first preclinical in vivo results of DARA in mouse models of mantle cell lymphoma, transformed follicular lymphoma and chronic lymphocytic leukemia has demonstrated strong ADCC and CDC activity in immunocompromised hosts. ${ }^{56}$ In this study, DARA induced complete tumor regression in four out of six mantle cell lymphoma mice and $60 \%$ tumor growth reduction compared to controls in transformed follicular lymphoma mice. In the case of chronic lymphocytic leukemia, it was shown that DARA not only increased survival by approximately threefold (limited mainly by the end of the preclinical trial) but also that it inhibited the homing and migration of leukemia cells from peripheral blood to bone marrow and spleen.

\section{Conclusion}

Traditional treatments including alkylating agents such as melphalan as well as newer proteasome inhibitor and immunomodulatory therapies such as BORT and LEN have demonstrated significant survival benefits as frontline agents in MM. Despite this, MM still remains a chronic and aggressive neoplasm with inevitable relapse.

The use of monoclonal antibody therapy in MM remains relatively nascent. ELO and milatuzumab have been under close investigation and have shown significant antitumor activity with potential application as both mono- or combination therapy. DARA is a humanized antibody to CD38 that has shown great promise as an effective monoclonal antibody with ADCC, CDC, ADCP, and direct cytotoxicity as its mechanisms of action. DARA also targets several vital cell-signaling pathways (including calcium messaging, T/B cell cross-talk, and NK cell activation) via inhibition of cyclic adenosine diphosphate-ribose and nicotinic acid adenine dinucleotide phosphate metabolism. In its first Phase I/II clinical study as monotherapy, dose-escalated DARA resulted in significant serum M-protein as well as bone marrow plasma cell reduction in a heavily pre-treated RRMM population. Toxicity was mainly infusion-related and mitigated by the 
use of pre-treatment steroids. Moreover, recent ex vivo studies have demonstrated that the addition of DARA to several existing regimens increases myeloma cell lysis. There is also preclinical evidence that DARA in combination with targeted radio-immunotherapy may have the potential to achieve high remission rates and that its monotherapeutic and/or combinatorial use in other hematologic malignancies may be vital.

Given the preliminary evidence for the use of DARA, there is great promise in its addition to the armamentarium for the treatment of RRMM.

\section{Disclosure}

Y Khagi has no conflicts of interest to declare. TM Mark: Speakers Bureau, Celgene, Millennium, Onyx; Research Funding: Celgene, Onyx; Advisory Board Member: Celgene, Millennium.

\section{References}

1. National Cancer Institute. SEER Cancer Statistics Review, 1975-2009 (Vintage 2009 Populations). Available from: http://seer.cancer.gov/ csr/1975_2009_pops09/. Accessed May 22, 2014.

2. Jemal A, Siegel R, Ward E, Murray T, Xu J, Thun MJ. Cancer statistics, 2007. CA Cancer J Clin. 2007;57:43-66.

3. Kyle RA, Rajkumar SV. Multiple Myeloma. $N$ Engl J Med. 2004;351(18):1860-1873.

4. Kumar SK, Rajkumar SV, Dispenzieri A, et al. Improved survival in multiple myeloma and the impact of novel therapies. Blood. 2008;111(5):2516-2520.

5. Kumar SK, Lee JH, Lahuerta JJ, et al. Risk of progression and survival in multiple myeloma relapsing after therapy with IMiDs and bortezomib: a multicenter international myeloma working group study. Leukemia 2012;26(1):149-157.

6. US Food and Drug Administration. Carfilzomib. Available from: http://www.fda.gov/Drugs/InformationOnDrugs/ApprovedDrugs/ ucm312945.htm. Accessed May 22, 2014.

7. US Food and Drug Administration. Pomalidomide. Available from: http://www.fda.gov/Drugs/InformationOnDrugs/ApprovedDrugs/ ucm339286.htm. Accessed May 22, 2014.

8. Quach H, Ritchie D, Stewart AK, et al. Mechanism of action of immunomodulatory drugs (IMiDs) in multiple myeloma. Leukemia. 2010;24(1):22-32.

9. Alsina M, Trudel S, Furman RR, et al. A phase I single-agent study of twice-weekly consecutive-day dosing of the proteasome inhibitor carfilzomib in patients with relapsed or refractory multiple myeloma or lymphoma. Clin Cancer Res. 2012;18(17):4830-4840.

10. Vij R, Siegel DS, Jagannath S, et al. An open-label, single-arm, phase 2 study of single-agent carfilzomib in patients with relapsed and/or refractory multiple myeloma who have been previously treated with bortezomib. Br J Haematol. 2012;158(6):739-748.

11. O'Connor OA, Stewart AK, Vallone M, et al. A phase 1 dose escalation study of the safety and pharmacokinetics of the novel proteasome inhibitor carfilzomib (PR-171) in patients with hematologic malignancies. Clin Cancer Res. 2009;15(22):7085-7091.

12. San Miguel J, Weisel K, Moreau P, et al. Pomalidomide plus low-dose dexamethasone versus high-dose dexamethasone alone in patients with relapsed and refractory multiple myeloma (MM-003): a randomized open-label phase 3 trial. Lancet Oncol. 2013;14(11):1055-1066.

13. Zojer N, Kirchbacher K, Vesely M, et al. Rituximab treatment provides no clinical benefit in patients with pretreated advanced multiple myeloma. Leuk Lymphoma. 2006;47(6):1103-1109.
14. Treon SP, Pilarski LM, Belch AR, et al. CD20-directed serotherapy in patients with multiple myeloma: biologic considerations and therapeutic applications. J Immunother. 2002;25(1):72-81.

15. Ngo NT, Brodie C, Giles C, et al. The significance of tumour cell immunophenotype in myeloma and its impact on clinical outcome. J Clin Pathol. 2009;62(11):1009-1015.

16. Moreau P, Voillat L, Benboukher L, et al. Rituximab in CD20 positive multiple myeloma. Leukemia. 2007;21:835-836.

17. Jakubowiak AJ, Benson DM, Bensinger W, et al. Phase I trial of anti-CS1 monoclonal antibody elotuzumab in combination with bortezomib in the treatment of relapsed/refractory multiple myeloma. J Clin Oncol. 2012;30(16):1960-1965.

18. Mark T, Martin P, Leonard JP, Niesvizky R. Milatuzumab: a promising new agent for the treatment of lymphoid malignancies. Expert Opin Investig Drugs. 2009;18(1):99-104.

19. Lonial S, Jagannath S, Moreau P, et al. Phase (Ph) I/II study of Elotuzumab (Elo) plus lenalinomide/dexamethasone (Len/Dex) in relapsed/refractory multiple myeloma (RR MM): Updated $\mathrm{Ph}$ II results and $\mathrm{Ph} \mathrm{I} / \mathrm{II}$ long-term safety. J Clin Oncol. 2013;31:(Suppl; abstr 8542).

20. Richardson PG, Jagannath S, Moreau P, et al. A phase 2 study of elotuzumab in combination with lenalinomide and low-dose dexamethasone in patients with relapsed/refractory multiple myeloma: updated results. Blood. 2012;120: Abstract 202.

21. Zonder JA, Mohrbacher AF, Singhal S, et al. A phase 1 multicenter, open-label, dose escalation study of elotuzumab in patients with advanced multiple myeloma. Blood. 2012;120(3):552-559.

22. Stein R, Mattes MJ, Cardillo TM, et al. CD74: a new candidate target for the immunotherapy of B-cell neoplasms. Clin Cancer Res. 2007; 13(18 Pt 2):5556s-5563s.

23. Burton JD, Ely S, Reddy PK, et al. CD74 is expressed by multiple myeloma and is a promising target for therapy. Clin Cancer Res. 2004;10(19):6606-6611.

24. Stein R, Qu Z, Cardillo TM, et al. Antiproliferative activity of a humanized anti-CD74 monoclonal antibody, hLL1, on B-cell malignancies. Blood. 2004;104(12):3705-3711.

25. Chang CH, Sapra P, Vanama SS, et al. Effective therapy of human lymphoma xenografts with a novel recombinant ribonuclease/ anti-CD74 humanized IgG4 antibody immunotoxin. Blood. 2005;106(13):4308-4314.

26. Griffiths GL, Mattes MJ, Stein R, et al. Cure of SCID mice bearing human B-lymphoma xenografts by an anti-CD74 antibody-anthracycline drug conjugate. Clin Cancer Res. 2003;9(17):6567-6571.

27. Sapra P, Stein R, Pickett J, et al. Anti-CD74 antibody-doxorubicin conjugate, IMMU-110, in a human multiple myeloma xenograft and in monkeys. Clin Cancer Res. 2005;11(14):5257-5264.

28. Lundberg BB, Griffiths G, Hansen HJ. Cellular association and cytotoxicity of anti-CD74-targeted lipid drug-carriers in B lymphoma cells. J Control Release. 2004;94(1):155-161.

29. Govindan SV, Goldenberg DM, Elsamra SE, et al. Radionuclides linked to a CD74 antibody as therapeutic agents for B-cell lymphoma: comparison of Auger electron emitters with beta-particle emitters. J Nucl Med. 2000;41(12):2089-2097.

30. Sapra P, Chang CH, Vanama S, et al. Preclinical Safety and Efficacy of Two Novel Immunotoxins Consisting of Ranpirnase (Rap) Fused to an Internalizing Anti-CD74 Humanized IgG4 Antibody in Human Non-Hodgkin's Lymphoma Xenografts. Proceedings of the American Society of Hematology Meeting 2005. Blood. 2005;106: abstract 346.

31. Kaufman JL, Niesvizky R, Stadtmauer EA, et al. Phase I, multicentre, dose-escalation trial of monotherapy with milatuzumab (humanized anti-CD74 monoclonal antibody) in relapsed or refractory multiple myeloma. Br J Haematol. 2013;163(4):478-486.

32. Noort WA, Groen RWJ, Raymakers R, et al. Daratumumab, a Novel Therapeutic Human CD38 Monoclonal Antibody, Induces Killing of Refractory Patient-Derived Multiple Myeloma Cells, Growing in a Novel Humanized Mouse MM Model. American Society of Hematology Proceedings. Blood. 2012;120: Abstract 940. 
33. De Weers M, Tai YT, van der Veer MS, et al. Daratumumab, a novel therapeutic human CD38 monoclonal antibody, induces killing of multiple myeloma and other hematological tumors. J Immunol. 2011;186(3): 1840-1848.

34. Lokhorst HM, Plesner T, Gimsing P, et al. Phase I/II dose-escalation study of Daratumumab in patients with relapsed or refractory multiple myeloma. J Clin Oncol. 2013;31(Suppl; abstr 8512).

35. Lin P, Owens R, Tricot G, Wilson CS. Flow cytometric immunophenotypic analysis of 306 cases of multiple myeloma. $\mathrm{Am} \mathrm{J}$ Clin Pathol. 2004;121(4):482-488.

36. Kim D, Park CY, Medeiros BC, Weissman IL. CD19-CD45 low/-CD38 high/CD138+ plasma cells enrich for human tumorigenic myeloma cells. Leukemia. 2012;26(12):2530-2537.

37. Hosen N. Multiple myeloma-initiating cells. Int J Hematol. 2013;97(3): 306-312.

38. Deaglio S, Mehta K, Malavasi F. Human CD38: a (r)evolutionary story of enzymes and receptors. Leuk Res. 2001;25(1):1-12.

39. Aarhus R, Graeff RM, Dickey DM, Waseth TF, Lee HC. ADPribosyl cyclase and CD38 catalyze the synthesis of calcium-mobilizing metabolite from NADP. J Biol Chem. 1995;270(51):30327-30333.

40. Lee HC. Structure and enzymatic functions of human CD38. Mol Med. 2006;12(11-12):317-323.

41. Deaglio S, Vaisitti T, Bergui L, et al. CD38 and CD100 lead a network of surface receptors relaying positive signals for B-CLL growth and survival. Blood. 2005;105(8):3042-3050.

42. Deaglio S, Vaisitti T, Aydin S, Ferrero E, Malavasi F. In-tandem insight from basic science combined with clinical research: CD38 as both marker and key component of the pathogenetic network underlying chronic lymphocytic leukemia. Blood. 2006;108(4):1135-1144.

43. Ferretti E, Bertolotto M, Deaglio S, et al. A novel role of CX3CR1/ CX3CL1 system in the cross-talk between chronic lymphocytic leukemia cells and tumor microenvironment. Leukemia. 2011;25(8): $1268-1277$.

44. Matas-Cespedes A, Vidal-Crespo A, Rodriguez V, Roue G, et al. Daratumumab, A Novel Human Anti-CD38 Monoclonal Antibody for the Treatment of Chronic Lymphocytic Leukemia and B-Cell NonHodgkin's Lymphoma. American Society of Hematology Proceedings 2012. Blood. 2012;120(21): Abstract 3935.

45. Jansen JH, Boross P, Overdijk MB, van Bueren JJ, Parren PW, Leusen JH. Daratumumab, a Human CD38 Antibody Induces Apoptosis of Myeloma Tumor Cells Via Fc Receptor-Mediated Crosslinking. American Society of Hematology Proceedings 2012. Blood. 2012;120(21):2974

46. Overdijk MB, Verploegen S, Marijn B, van Egmond M, Growen RW, Anton CM. Martens, et al. Phagocytosis is a Mechanism of Action for Daratumumab. American Society of Hematology Proceedings 2012. Blood. 2012;120(21):4054.
47. Van der Veer MS, de Weers M, van Kessel B, et al. The therapeutic human CD38 antibody daratumumab improves the anti-myeloma effect of newly emerging multi-drug therapies. Blood Cancer J. 2011;1(10):e41.

48. Kong SY, Li XF, Nahar S, Song W, de Weers M, Parren PW, et al. Daratumumab Directly Induces Human Multiple Myeloma Cell Death and Acts Synergistically with Conventional and Novel Anti-Myeloma Drugs. American Society of Hematology Proceedings 2010. Blood. 2012;116(21):3013.

49. Van der Veer MS, de Weers M, van Kessel B, et al. Towards effective immunotherapy of myeloma: enhanced elimination of myeloma cells by combination of lenalidomide with human CD38 monoclonal antibody daratumumab. Haematologica. 2011;96(2):284-290.

50. Plesner T, Lokhorst H, Gimsing P, Nahi H, Lisby S, Richardson PG. Daratumumab, a CD38 Monoclonal Antibody in Patients with Multiple Myeloma- Data from a Dose-Escalation Phase I/II Study. American Society of Hematology Proceedings 2012. Blood. 2012;120(21):73.

51. Plesner T, Lokhorst HM, Gimsing P, Nahi H, Lisby S, Richardson PG. Daratumumab, a CD38 mab, for the treatment of relapsed/refractory multiple myeloma patients: Preliminary Efficacy Data from Multicenter Phase I/II Study. J Clin Oncol. 2012;30(Suppl; abstr 8019).

52. Janssen Research and Development, LLC. An Efficacy and Safety Study of Daratumumab in Patients With Multiple Myeloma Who Have Received at Least 3 Prior Lines of Therapy (Including a Proteasome Inhibitor [PI] and Immunomodulatory Drug [IMiD]) or Are Double Refractory to a PI and an IMiD. Available from: http://clinicaltrials.gov/show/NCT01985126. NLM identifier: NCT01985126. Accessed May 22, 2014.

53. Nijhof I, Noort WA, Van Bueren JL, et al. CD38-Targeted Immunochemotherapy of Multiple Myeloma: Preclinical Evidence for Its Combinatorial Use in Lenalinomide and Bortezomib Refractory/ Intolerant MM Patients. Proceedings of the American Society of Hematology 2013. Blood. 2013;(122):277.

54. Plesner T, Arkenau T, Lokhorst H, et al. Preliminary Safety and Efficacy Data of Daratumumab in Combination with Lenalinomide and Dexamethasone in Relapsed or Refractory Multiple Myeloma. Proceedings of the American Society of Hematology 2013. Blood. 2013;122:1986.

55. Green D, Jones J, Hylarides MD, et al. Anti-CD38 Pretargeted Radioimmunotherapy Eradicates Multiple Myeloma Xenografts in a Murine Model. Proceedings of the American Society of Hematology 2013. Blood. 2013;122:882.

56. Matas-Cespedes A, Crespo AV, Rodriguez V, et al. Daratumumab, A Novel Human Anti-CD38 Monoclonal Antibody Shows Anti-Tumor Activity in Mouse Models of MCL, FL and CLL. Proceedings of the American Society of Hematology 2013. Blood. 2013;122:378.
OncoTargets and Therapy

\section{Publish your work in this journal}

OncoTargets and Therapy is an international, peer-reviewed, open access journal focusing on the pathological basis of all cancers, potential targets for therapy and treatment protocols employed to improve the management of cancer patients. The journal also focuses on the impact of management programs and new therapeutic agents and protocols on
Dovepress

patient perspectives such as quality of life, adherence and satisfaction The manuscript management system is completely online and includes a very quick and fair peer-review system, which is all easy to use. Visit http://www.dovepress.com/testimonials.php to read real quotes from published authors. 